\title{
Interrogantes éticos subyacentes al problema del empleo
}

\author{
Por el Prof. Jean Claude SALLY.
}

\begin{abstract}
En la primera parte se interroga sobre la manera de ela. borar un procedimiento de reflexión ética acerca de los problemas económicos. Luego se aplica este procedimiento a la cuestión del empleo y del paro.
\end{abstract}

\begin{abstract}
A nivel económico, los problemas provocados por el empleo y el paro a finales del siglo $X X$ son terribles: los economistas se dividen en dos grupos, uno dedicado a analizarlos y otro dedicado a hallar los remedios que deben aportarse. En el plano ético, cada uno adivina la importancia de los problemas que ocasiona la crisis económica mundial, en especial en sus repercusiones sobre el empleo; pero los moralistas apenas se atreven a formular un diagnóstico ni a proporcionar una clave que ayude a los agences económicos y sociales a aclarar su análisis y a tomar sus decisiones. Por tanto, si economistas y moralistas, cada uno dentro de su disciplina, se muestran discretos y reservados en este punto, ¿cuál no será la proeza de intentar una reflexión que integre los enfoques del economista y del moralista sobre el empleo? Este intento es tanto más difícil cuanto que se introduce de forma bastante particular en el movimiento de este congreso; el presente informe, redactado antes del congreso debe ser presentado al final de éste, es decir, en un momento en que se hayan aportado diversas aclaraciones, en especial de tipo económico, pero también de orden ético, lo cual deberá modificar algunas de las cuestiones presentadas más abajo. De este modo, la formulación actual del problema corre el riesgo de ser caduca, incluso para nosotros, cuando tengamos que presentarla a los participantes del congreso. Así, para escapar en parte a esta dificultad, el objetivo que se ha fijado en las páginas siguientes es el de plantear los jalones necesarios para la elaboración de un procedimiento de reflexión más bien que el llegar a formulaciones éticas propiamente dichas, lo que debería constituir uno de los productos de otras sesiones del congreso.
\end{abstract}

Las consideraciones que siguen son el fruto de una reflexión común (1) Ile-

"Encargado de Investigaciones en el C. N. R. S. profesor en la Facultad Libre de Clencias Económicas de Lille y en el Instiuto de Economía Clen. túfica y de Gestión, director del Centro de Investigaciones Económicas, Soclológicas y de Gestión.

(1) La parte ética de esta exposición debe muchos puntos a las reflexiones de $C$ H. LEFEVRE, G. MATHON y J. MOUSSE; la parte econónica a la aportación de M. FALISE. 
vada a efecto dentro del marco del Centro Interdisciplnario de LLLE con economistas, moralistas y teólogos. Han sido especiamente elaboradas con ocasión de la celebración en LltuE, en septiembre de 1980, de un congreso de moralistas y de teólogos sobre el problema del empleo. Sin embargo, el autor asume toda la responsabilidad de las formulaciones que propone dentro del marco de este estudio.

En la primera parte, ampliando el problema del empleo a una dimension más general, reflexionaremos sobre la manera de elaborar un procedimiento de reflexicn etica acerca de los problemas económicos. Luego, aplicarenos este procedimiento a la cuestión del empleo y del paro, tanto a nivel de cuestionarios recurriendo a la observación de hechos (punto II) como al de interrogantes más propiamente éticos (punto III).

\section{ELABORACION DE UN PROCEDMENTO DE REFLEXION}

Pueden concebirse varios enfoques para poder llevar a cabo una reflexión ética sobre los problemas económicos. Ante todo, presentaremos un procedimiento que nos parece poco fecundo y cuya crítica nos permitirá formar - abrir un camino que creemos, es más conforme con la naturaleza del problema que tenemos que estudiar.

1.1. Una primera concepción supone cierta visión de la clencia económica, de la moral y de las relaciones que deben tejerse entre estas dos disciplinas. En esta perspectiva, la gestión consistira, por una parte, en plantear un razonamiento de tipo cientifico dentro del orden económico. Podría efectuarse una lectura únicamente objetiva de los hechos económicos, pudiendo escaparse esta lectura a los condicionamientos humanos y sociales de los que la hacen y pudiendo desprender cierto consenso sobre la veracidad de los análisis realizados y sobre el abanico de soluciones proponibles, consenso al que deberian adherirse todos los cientificos serios y de buena fe: dicho de otro modo, seria posible ponerse de acuerdo sobre un análisis cientifico de las realidades humanas. A este trabajo efectuado por los economistas correspondería, por otra parte, una reflexión iniciada esta vez por los moralistas. Sobre estos recaería la tarea de establecer un cuerpo de valores susceptible de proporcionar al conjunto de los hombres caiterios para sus decisiones y sus acciones. Correspondería entonces a todos aquellos que deseen vivir sus propias elecciones de forma moral (cientificos, pero también hombres de acción. sea cual sea sif responsabilidad integrar estos dos universos para interpretar convenientemente las realidades y para orientar su conducta en el sentido deseado: unión lograda de la lucidez y de los grandes principios.

1.2. Esta presentación, por supuesto endurecida por las necesidades del estudio, de las relaciones entre economia y moral, suscita muchas cuestiones.

Ante todo, a nivel de la concepción de la economia política. Ciertamente, esta es una gestión de tipo científico y, por ello, tiene por ambición, en su orden epistemológico, medir y explicar objetivamente los fenómenos económicos. Sin embargo, es preciso considerar que es una ciencia humana, lo cual tiene dos consecuencias para nuestro propósito. En primer lugar, el objeto mismo de su conocimiento, el hombre enfrentado con la producción de su exis. tencia, es un ser de libertad, aunque las elecciones morales de este último influyan en el mundo económico que él construye. Además, esta libertad se ejerce en un entomo que, a la vez, es su fruto y lo condiciona, aungue las 


\section{INTERROGANTES ETICOS}

"leyes economicas" esten siempre situadas en un contexto que incluye, al primer vistazo, una dimensión fitica. En segundo lugar, la realidad economica que es preciso adoptar para llevar a cabo un trabajo cientifico es demasiado compleja y demasiado dispersa para poder ser aprehendida en su totafidad. Por ello, no existe ninguna lectura cientifica que sea exhaustiva $y$, por tanto, verdadera, y por lo mismo imponible a todos los actores racionales (aum cuando se pueda progresar en la calidad de las diversas teorias existentes) Esto es tanto más cierto cuanto que cada clentifico, marcado socialmente, tiene tendencia, incluso si trata de defenderse, a abordar to real descie su punto de vista. Así, ya no se asombra uno, a propósito de los problemas de empleo, así como de otros, al comprobar diferencias de análisis bastante radicales de un experto a otro.

Un segundo criterio que hay que aportar al trabajo anteriormente planteado se refiere al concepto de la moral. ¿Es posible definir un cuerpo de valores que el conjunto de los actores podría adoptar y a la luz del mismo organizar su acción? La realidad es menos sencilla. Ante todo, porque los valores son antitéticos. Privilegiar uno significa que se entra en conflicto con el otro: cada uno de nosotros ha reflexionado en las parejas de valores, cuyo avance más frecuente en economía es el tándem eficacia-solldaridad. Por tanto, si los valores se contrarian, son posibles muchos sistemas de valores. ¿Cuál elegir y erigir como cuerpo de valor propuesto para todos? ¿En nombre de qué criterios efectuar esta elección?

Un acercamiento sociológico muestra que las jerarquías de valores difieren de una sociedad a otra, de un grupo social a otro: jcuál podría ser el punto de vista de Sirius que estuviera en condiciones de dar un moralista imparclal? ¿No existe ninguno! Tanto más que $(y$ es la segunda razón que comprueba la complejidad de las cosas) no hay elección moral más que la enraizada en lo concreto. Los valores son vividos en la complejidad de la vida económica: se traducen en las estructuras económicas, en el sistema institucional, en los objetos fabricados; están ya operando en el mundo en gestación y no se trata de hacerlos bajar de un mundo de ideas para informar de una realidad gastada que les sería extraña antes de que se introdujeran asi.

Se ve, pues, lo poco fecundo que aparece a priori, el concepto de que. rer organizar una confrontación entre, por una parte, una ciencia económica únicamente técnica, que edifique en un consenso general las leyes económicas universales que se escapan de las discrepancias de puntos de vista e intereses y extraños, en su orden, a los problemas éticos $y$, por otra parte, una construcción moral que edifique en lo abstracto una jerarquía de valores que vendrá a alumbrar, en un segundo tiempo, la conciencia humana previamente instruida de los resultados de la ciencia.

13. Una vez hecha esta crítica, ¿qué procedimiento general proponemos? Nos parece que el diálogo de enfoque económicoreferencia a los valores debe ser más intimo y más permanente que en lo anteriormente expuesto. Ciertamente, conviene no confundir los dos enfoques (tienen su objetivo y sus procedimientos propios), pero hay que conjugarlos de manera más intima. Para ser concreto, nos parece que una reflexión técnica sobre los problemas del emples (pero igualmente sobre cualquier otra cuestión de tipo económico) deberia desarrollarse en tres etapas.

la primera etapa consistiría en examinar las dificultades y los grados de 


\section{J. C. SAILLY}

libertad de que disfrutan los diferentes actores en presencia. En efecto. solamente hay elección moral si hay posibilidad de eleccion simplemente $y$, por tanto, algún grado de libertad. Esto resulta naturalmente en gran parte de una lectura científica pero, si esta se comprende bien, incluye ya relaciones con los valores. Importa, en efecto, este análisis tenga en cuenta los elementos siguientes:

- la realidad que hay que estudiar es compleja: todos los puntos de vista resultan parciales, ninguno de ellos debe considerarse absoluto: hay ya una gestión de tipo moral (relación de valor a verdad) en esta relativización de conocimientos;

- los actores son múltiples, están situados a niveles de responsabilidad diferentes, están inscritos en las relaciones de fuerza que condicionan sus grados de libertad. Los espacios de autonomía y, por tanto, de elección moral, no son los mismos según la situación de los actores; además, las jerarquias de valores adoptadas difieren en función de este mismo criterio.

La segunda etapa tendría como objetivo localizar detrás de los diversos grados de libertad de que se benefician los actores, los valores que corren el riesgo de fortificarse 0 , por el contrario, de descuidarse en la elección de las diversas soluciones hechas posibles por estas zonas de libertad. Aqui todavía se observa una estrecha imbricación entre los análisis de tipo cientifico y los juicios de valor: en función de las consecuencias económicas de tal comportamiento de un actor, tal valor que estará fortalecido por este útumo será por el contrario menos válido para cualquier otro asoclado: las conse. cuencias de una decisión tendrán repercusiones muy diferentes sobre tal valor según que se considera un plazo corto o largo, etc...

Finalmente, la tercera etapa consistiría en elaborar criterios de decisión para los actores. Deben distinguirse varios niveles: conviene establecer una batería de criterios nacida de la observación de las exigencias resultantes de toda vida de sociedad; se puede ir más lejos y poner en práctica un conjunto de criterios que se enraizan en una familia filosófica o religiosa; en toda hipótesis conviene relativizar las elecciones parciales y provisionales que finalmente serán adoptadas por los actores.

Como se ve, las dos primeras etapas surgen más bien de la observación de los hechos, pero se introducen ya en cierta gestión ética. La tercera recurre al moralista. Antes de realizar la aplicación de este procedimiento al problema del empleo, conviene precisar la actitud moral fundamental que debe caracterizar el recorrido de estas etapas así como los principios éticos susceptibles de aclarar las tomas de decisión.

1.4. Para cualficar la actitud moral que debe impregnar la gestión, es preciso recordar las características de la acción humana y, por tanto, de la acción moral que se han evocado anteriormente: se trata de intervenir en una realidad, compleja, intotalizable, fragmentada en múltiples aspectos en interacción; construida por numerosos autores, que presenta intereses diferentes, a menudo opuestos e inconciliables; se encuentra, en fin en un continuo pro* ceso de cambio. Estas particularidades requieren una actitud moral que se puede traducir en tres imperativos.

Si es cierto que el comportamiento ético nace de la posibilidad de alección y, por tanto, de los grados de libertad en la acción, conviene ante todo 
examinar con todo cuidado la naturaleza y la ampiitud de estas zonas de li. bertad $y$, por tanto, de responsabilidad. Para todos los actores, esta fase de análisis es determinante; a menudo se olvida o escamotea. ise ha hecho ver daderamente un inventario de las latitudes ofrecidas? ¿No se han apretado demasiado los tornillos de las dificultades y reducido el abanico de posibilidades? ¿Se ha tomado suficientemente conciencia de que las dificultades de hoy pueden hacerse variables mañana? De seguro, esta fase de examen serio y completo de datos forma ya parte del comportamiento ético. Condiciona in. cluso toda la fecundidad de etapas ulteriores.

Del carácter complejo y disperso de esta realidad que es preciso analizar con cuidado para descubrir en ella todas las potenciabilidades, nace el segun. do imperativo moral: este análisis debe ser también lo más completo posible $y$, sin embargo, debe permanecer consciente de su carácter necesariamente parcial. En razón de las múltiples interacciones entre lo económico, lo social, lo politico, lo institucional, lo legislativo, etc., importa tener un enfoque que trate de integrar el conjunto de estas perspectivas en el diagnóstico sobre las dificultades y los grados de libertad: la actitud moral exige, pues, un saber abierto. $Y$, por tanto, un saber que se sabe es limitado $y$, sin duda, parcial. Ello implica que se reconoce el carácter relativo de su punto de vista, que se acepta que hay otros, detectando igualmente su parte de verdad. Esta toma de conciencia prepara el advenimiento de compromisos que entonces pueden ser menos la traducción de relaciones de fuerza que el resultado de

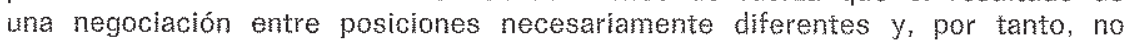
absolutas.

Esto lleva al tercer imperativo: si los análisis son parciales, si los puntos de vista son diversos, las mísmas opciones éticas fundamentales pueden traducirse en confrontaciones concretas entre actores y solamente pueden resolw verse con un compromiso. En esta perspectiva, la actitud moral necesita a la vez la acogida y la escucha de las posiciones de los demás, la firmeza con respecto de lo que se estima éticamente intangible (desde este punto de vista, la violencia puede ser utilizada para combatir otras violencias) y la búsqueda de compromisos que traduzcan la voluntad de vivir juntos. Estos com promisos son, por otra parte, provisionales; la actitud moral se apoya en la evolución constante de las situlaciones para volver a interrogar a las elecciones de ayer.

Una vez recordados los grandes principios del comportamiento moral, zqué conjunto de criterios éticos pueden ofrecerse dentro del marco de una opción personalista iluminada por la revelación cristiana?

El principio primero del que fluyen todos los demás podría ser el del reconocimiento mutuo: que cada uno reconozca al otro como sujeto y renuncie a todo espiritu de dominación así como a toda actitud servil. El desarrollo de este principio nos llevará más lejos: el problema es, en efecto, traducirlo a lo concreto a través del juego de las instituciones y la transformación de las cosas: que el funcionamiento de las instituciones favorezca la libertad de las personas y que la relación con las cosas no se convierta en un objetivo al cual esté subordinada cualquier relación con los demás.

Este principio fundamental está reforzado y desarrollado por la aportación ética que dé la revelación cristiana; puede citarse a este respecto: of rechazo a la idolatría, en particular la idolatría del poseer; la prioridad concedida a la calidad de la relación con otro, estando éste revestido de la dig- 


\section{J. C. SALLY}

nidad propia de Dios, sobre todo cuando es débil y despreciado; la importan cia reservada al reparto y a la desposesión, ya sea en el orden del poseer. del saber o del poder; el acento puesto en la valoración y la transfomación del universo con vistas al engrandecimiento de todo al hombre y todos los hombres.

Podrian desprenderse otros criterios de acción. Los que hemos citado son lo suficientemente exigentes como para estimular la reflexión ética, en particular en el problema que nos preocupa. En esta exposición no puede tratarse de desarrollar con todo el cuidado deseable las tres etapas evocadas anteriormente. Más explicitamente, se tratará de las dos primeras en la segunda parte de este estudio. La tercera se esbozará en el puhto III.

\section{GRADOS DE LIBERTAD Y VALORES EN CUESTION}

Si seguimos el procedimiento que preconizamos, conviene, pues, preguntarse sobre las dificultades, sobre las zonas de libertad (etapa 1) asi como sobre los valores que favorecen o desfavorecen los diversos comportamientos que pueden inscribirse en estos espacios de autonomía (etapa 2), y ello para cada uno de los grupos que intervienen en el problema del empleo. Los dos primeros días del congreso se dedicarán a esta interrogante, lo que deberá aportar toda clase de materiales nuevos a este cuestionario. Asimismo, en al estadio de este trabajo, nos contentaremos con redactar una primera lista no exhaustiva de los grados de libertad y de los valores subyacentes para tres categorías de asociados: los poderes públicos, las empresas y las organizaciones salariales. Esta primera base de discusión se ampliará, se enriquecerá y se corregirá, ciertamente, en los trabajos de las diversas sesiones consagradas al comportamiento de cada una de estas tres instituciones.

\section{II.1. Los poderes públicos}

Se analizarán tres tipos de acción:

\section{- La polifica macroeconómica que opera sobre el volumen de horas de-} mandadas:

Podrian examinarse varios elementos que influyen en este último parám metro: ¿cuáles son las dificultades y los grados de libertad en materia de determinaciôn del tipo de crecimiento del producto real, de la productividad media de las horas de trabajo, del volumen del gasto público? ¿Cuáles son los posibles escenarios (que se han estudiado)? ¿Cuál es la suma algebráica de sus efectos? ¿Se han explorado las diversas hipótesis imaginables? ¿Qué difusión se ha hecho de estas exploraciones?

Detrás de estas diferentes elecciones posibles lo detrás de las elecciones efectivas), zcuáles son los valores que son privilegiados: la afirmación de la independencia y de la potencia nacional; el mantenimiento o la elevación del nivel de vida (en término medio o para todos los ciudadanos); la investigación exclusiva de la competitividad o de cierto consenso social; la inves. tigacion real del pleno empleo o la aceptación de un volante de paro destinado a calmar las presiones inflacionistas de los salarios? 
- La politica macroeconómica de solidarided intramaclonal ante el problema del empleo:

¿De qué margen dispone el Estado en la repartición del paro entre categorias de individuos? ¿Deja desarrollar espontáneamente al juego de las rela* ciones de fuerza que probablemente tiende a colocar sobre los más débiles la carga del aumento de insegurided global -0 prosigue en la materia una politica dellberada de repartición correctiva? (Detrás de estos interrogantes surge la cuestión de la repartición del paro entre el sector público y el sector privado, entre el sector protegido y el sector abierto a la competencia internacional, entre empresas en dificultades pero con fuerte poder de negociación (como son las firmas siderúrgicas) y las que casi no disponen de triunios politicos, entre regiones dinámicas y zonas depresivas, etc.].

Cuando los poderes públicos deciden intervenir en esta repartición, ilo hacen principalmente jugando con los ingresos a través de las transferencias sociales? (y en este caso, ¿quién se beneficia de ello o quién, por el contrario, se escapa de ello?) o la emprenden así y directamente con el compo. nente empleo? En este último caso, ¿cuáles son los medios contemplados?

¿Qué valores están en juego detrás de estas elecciones posibles: preocupación exclusiva de eficacia o preocupación de solidaridad, etc.?

\section{- La política macroeconómica ante los países extranjeros:}

El problema del paro interior en nuestros paises está ligado al de nuestras relaciones económicas exteriores, especialmente bajo dos aspectos: modificación en las relaciones de fuerza internacionales fprecio de la energía, competencia de nuevos países industrializados\} e impacto sobre el paro del flujo de emigración de mano de obra. Aqui todavia, conviene identificar los grados de libertad de que se benefician los poderes públicos así como las escalas de valores correspondientes.

A título de llustración, ¿es una preoctpación únicamente nacionalista la que guía a los políticos con respecto de otros paises industrializados y de paises en vías de desarrollo? ¿Cuál es el lugar de la concertación internacional en la toma de decisión? Tanto en los problemas de la circulación de pro ductos como en los de la circulación de los hombres, ise preocupan de la solidaridad entre naciones y del porvenir de las diversas fracciones del mundo?

\subsection{Las empresas}

¿Cuál es el puesto del empleo en el conjunto de los objetivos de las firmas? Esto se traduce a la vez en la concepción de la empresa y en las prác ticas concretas que fluyen de ella:

\section{- El concepto general de la empresa:}

En el espiritu de sus dirigentes, ¿está la empresa concebida exclusivamente como una célula de producción de riqueza con, como solo criterio de valoracion. su cashthow o su cifra de negoclos? ¿ES vista igualmente como una célula sochal? Esto supone, por una parte, una responsabilidad social interna 


\section{J. C. SAILLY}

ante la mano de obra, su empleo, sus ingresos, sus condiciones de trabajo: esto implica, por otra parte. una responsabilidad social "ad extra" con respecto al entorno de la empresa, a través especialmente del empleo inducido que suscita. Si las acciones de la empresa suponen un impacto sobre este entorno. la firma ejerce, por tanto, una responsabilidad de tipo societario. Si estas responsabilidades sociales y societarias son reconocidas y se toman en cuenta, la función que reúne los objetivos de la empresa desborda los solos criterios del cashoflow y de la cifra de negocios, y estos últimos se convierten en objetivos zorzados y ya no en los únicos objetivos que hay que maximizar.

Detrás de estos diversos conceptos de la empresa están presentes jerarquías de valor muy diferentes que conciernen al puesto de lo económico en lo social, al papel de la empresa y a la función de los dirigentes, al papel del beneficio, a la responsabilidad conjunta de los jefes de empresa en una zona de empleo, etc.

\section{- Las prácticas concretas ante el empleo:}

El tipo de concepción retenida-puramente económica o igualmente social, esto es, societaria-se traduce en cierto número de comportamientos, de los que se enumerarán los más importantes.

¿Cuál es el lugar reservado a la información y a la participación de los asalariados en las decisiones? ¿De forma habitual? ¿En caso de amenaza de despidos?

¿Cuál es la política de localización de las inversiones?

En la politica de personal, ise tiene preocupación prioritaria por «enflaquecer" y descartar a los menos aptos o se tiene preocupación simultánea de "desarrollar la musculatura", es decir, promover y formar, incluidos los menos bien provistos?

¿Qué iniciativa se tiene ante nuevas formas de empleo que permiten una mejor distribución: horarios flexibles, trabajo por horas, etc.? En la hipótesis de una reducción de la duración del trabajo, globalmente, de una estabilización, aun de una amputación de los ingresos reales, iqué opciones se toman referentes a la repartición entre asalariados de esta estabilización o de esta reducción del poder de compra y referentes a la posibilidad para el personal de compensar estos efectos negativos de ingresos por una mejora de las condiciones de trabajo?

\section{Il.3. Organizaciones salariales}

Merecen explorarse tres tipos de estrategias:

\section{- Las estrategias de defensa del empleo:}

¿Cuál os el objetivo de estas estrategias: llegar realmente o abocar real. mente a salvar el empleo o, simultáneamente, asegurarse una fidelidad de la base, mantener un mínimo de disputas sociales el función de objetivos polín ticos, etc.? 
¿Cuáles son los beneficios de estas estrategias: la totalidad de los em. pleos amenazados o las categorias más potentes, y esto en detrimento de los débiles? El efecto de estas estrategias, ino es parciatmente fomentar el mercado paralelo o inseguro del empleo? En estas estrategias, ¿cuál es el lugar reservado a los asalariados de otras naciones, en particular a los de los paí ses en vías de desarrollo?

\section{- Estrategias con respecto a nuevas formas de empleo:}

¿Cuáles son los grados de libertad y las escalas de valor subyacentes en lo que afecta a las fórmulas de trabajo por horas, horarios flexibles, ordenar ción progresiva de la jubilación, empleo más intensivo de las máquinas...?

\section{- Estrategias con respecto a problemas más globales:}

Clertas reivindicaciones tradicionales en materia especialmente de ingresos de resistencia a nuevas formas de trabajo, ino ponen en peligro el empleo, bien sea directamente a nivel de empresa, bien indirectamente a nivel de la nación a través de un aumento de cargas sin contrapartida de eficiencia? ¿Qué conciencia existe de este tipo de incidencias y qué responsabilidades se asumen a este respecto por las organizaciones salariales? ¿Cuál es la legitimidad de ciertas huelgas y reivindicaciones?

Tales son algunos de los interrogantes más importantes que permiten encuadrar las zonas de libertad de que se benefician los actores en presencia. Detras de estas posibilidades de elección, se revelan conjuntos de valores. Conviene tratar de progresar en la reflexión ética.

\section{HACIA LA ADOPCION DE UN JUICIO ETICO}

Esta tercera parte solamente será embrionaria, ya que también debería estar enriquecida por las sesiones del congreso. Se querría sintetizar el conjunto de las cuestiones que provoca el examen de los grados de libertad y de los valores que aquellos ponen en práctica y esto a la luz de los principios elaborados al comienzo de la exposición.

111.1. En primer lugar, es preciso relativizar la cuestión del empleo. Quiere decirse con ello que éste no es un objetivo en sí, como tampoco lo es el cre. cimiento por sí mismo. La prioridad no es que todos tengan un trabajo, sino que los hombres inventen un vivir juntos que facilite el reconocimiento mutuo. En esta perspectiva, el trabajo se convierte en un medio.

A este respecto, es iluminador distinguir varias significaciones en el trabajo. Este último permite ante todo la cobertura de las necesidades (trabajo-necesidad). Autoriza después la realización de la persona en la confrontación con la naturaleza y los demás (trabajo-obra). Sitúa finalmente al trabajador en un mundo de relaciones en la construcción del mundo con otros.

En el interrogante sobre los problemas del empleo y sobre las soluciones aportadas al paro, conviene poner de manifiesto estos tres objetivos del trabajo, lo que implica un cuestionario sobre el funcionamiento global de la socledad: 
J. C. SAILLY

-.-. zno hay tendencia a privilegir el primer aspecto en detrimento de los otros dos?

-.- los mecanismos de reconocimiento social, ¿no están ligados esencial. mente al ejercicio de un trabajo remunerado (un empleol y al nivel de ingresos al que da derecho?

Estas reflexiones generales desombocan en interrogantes particulares:

- referentes a la garantía de recursos:

- ipuede contentarse uno en contemplar úncamenta este aspecto en la soluctón del problema del paro?;

- con respecto de la noción de solidaridad y de distribuciór ise plan. táa el problema de la misma manera que para el conjunto de los agentes económicos: fcuestión de una cierta proporcionalidad entre indemnizaciones de paro y salario anterior; cuestión del segundo salario en una familia de condición acomodadal?;

* referente a la inserción social en la participación en una obra colectiva:

- iqué progresos realizar en las condiciones de trabajo para que se afirme an más, a nivel de mayor número, esta conciencia de colaboración en una obra común?;

- ¿cómo condicionar la significación del trabajo-obra con ciertos efectos del progreso técnico sobre el contenido de las tareas?;

- con respecto a la noción de solidaridad, ipara quién es más indispensable el reconocimiento social por el empleo?:

- empleo remunerado, ies la unica forma de reconocimiento social? ¿No se puede imaginar para algunos ciertas formas tan florecientes rehabilitación de la benevolencia; revalorización de la condición social, de la educadora o del educador de jóvenes en el hogarl?

- referente a la unión entre empleo, ingresos y promoción de las personas:

- ¿no es deseable desconectar más calidad de la vida y promoción humana, por una parte, tiempo de empleo remunerado e ingresos reales. por otra, lo que vuelve a poner en litigio el lugar central concedido en nuestras sociedades al nivel de vida cuantitativo?

Es sin duda a la luz de esta cuestión como deben examinarse las formas de una distribución ampliada del empleo trabajo por horas més bien que toda la jornada, jubitación anticipada acompañada por una reduccion de ingresos, limitación del pluriempleo.

111.2. Es a la luz del principio de reconocimiento mutuo aplicado a las situaciones concretas (especialmente las relaciones de fuerzas existentes). como deben arbitrarse los diversos conflictos de valores que el punto II de esta exposición ha señalado: se evocarán especialmente los conflictos entre solidaridad local (regional o nacional) y solidaridad internacional, entre seguridad y adaptabilidad, entre responsabilidad, exclusivamente económica y responsabilidad social y societaria de la empresa, entre solidaridad y eficacia.

Cada situacion merece un examen particular, habida cuenta de las posturas $y$ de las relaciones de fuerza, para que un juicho ético pueda aportarse. Nos contenaremos con evocar aqui este a aquel ejemplo que indica el proce 
dimiento a seguir. Asi, la seguridad total en materia de empleo va contra la exigencia de adaptabilidad y de movilidad tecricamente necesaria en una economia, exigencia que puede ser iqualmente portadora, en ciertas condiciones, de potencial de promoción humana. El examen de las relaciones de fuerza, ¿no deberia conducir a reservar esta seguridad de empleo a los que son naturalmente los menos adaptables (personas de edad, incapacitados, personal menos cuatificado), lo que vendría de nuevo a desasegurar a los fuertes para asegurar nejor los débiles?

Iguamente, se puede concebir que en los conflictos entre intereses locales e intereses mayores sea preciso conciliar la necesaria abertura a lo universal y los niveles de responsabilidad de cada uno: un jefe de emoresa textil debe tener en cuenta, con toda certeza, el desarrollo industrial da los NPI, en particular en la perspectiva de la expansión de su firma pero es especiamente dal porvenir de sus asalariados de lo que se debe preocupar: en el Estado la mayor preocupación es redefinir con los otros paises las reglas del juego de una colaboración internacional más solidaria.

Para cada tipo de problema ligado con el empleo, la gestión ética ve así a cada asociado situado dentro de su grupo y con respecto de los demás grupos, conociendo los grados de libertad de que dispone y consciente de la parcialidad de su punto de vista, volviendo a poner en duda sus comportar mientos y sus costumbres y abierto a las posiciones de los competidores o de los adversarios. Las opciones y las decisiones se hacen en una semiclafidad y abocan a compromisos provisionales: para quien reflexiona sobre las interrogantes éticas subyacentes a los probiemas económicos, la luz sorda, incierta, de cuestionar incesantemente la acción y los ackores.

Al término de esta exposición, cabe preguntarse sobre las responsabiltdades particulares de nuestras instituciones, de nuestros profesores y cuadros con respecto a las ideas desarrolladas en el presente informe: subrayaremos solamente tres puntos que nos parecen importantes.

Nuestra primera responsabilidad es ciertamente la de ser buenos científicos $y$, por tanto, colaborar eficazmente en la construcción de un razonamien. to cientifico pertinente. Acerca de este punto, si lo que hemos dicho de la naturalezá de la ciencia económica como ciencia humana es exacto, zno cabe el peligro de encerrar la realidad en una lectura que tendríamos tendencia a creer exhaustiva? ¿Cuál es el lugar que se ha concedido a lo social y a to sociológico en el entomo de nuestros análisis económicos?

Si es clerto que la acción moral se hace posible por el descubrimiento de grados de libertad, lo cual resulta de un análisis objetivo, la función que los cientificos deben cumplir es determinante, desde ese punto de vista. ¿Qué papel representamos en el marco de nuestros estudios personales, en el marco de las consultas que concedemos o que nos piden, en la puesta en evidencia de esas zonas de libertad? ¿En qué tipos de actores nos interesamos más particularmente? ¿Qué lugar ocupa el problema del empleo (a nivel de nuestra región o de nuestro pais) en nuestras preocupaciones?

Finalmente, puesto que formamos futuros cuadros que tendrăn que ejercer responsabilidades economicas, ¿qué lugar se da en su formación científica y humano a la integración de algunas llamadas evocadas mas arriba y que con- 


\section{J. C. SAILLY}

dicionan las tomes de decision (la realidad ha estallado los actores son diversos y parciales (incluidos nosotros mismos!)? Conviene situar exactamente las dificultades y los márgenes de actuación. Un objetivo todavia más ambjcioso: ¿qué reflexion se les propone para implicar las posturas éticas en las decisiones que tendrán que tomar?

Para cada uno de nosotros, dentro de nuestras instituciones, marcadas por una historia, relaciones de fuerza, costumbres, hombres, en el lugar que ocupamos, se abren zonas de libertad que ofrecen la posibilidad de desviar los comportamientos. ¿Sabremos descubrirlas e inventarlas?

Lille, 26 de marzo de 1981 